\title{
Low-Dimensional Carbon Nanomaterials: Synthesis, Properties, and Applications
}

\author{
Sulin Zhang, ${ }^{1}$ Teng Li, ${ }^{2}$ Jianyu Huang, ${ }^{3}$ and Vivek Shenoy ${ }^{4}$ \\ ${ }^{1}$ Department of Engineering Science and Mechanics, The Pennsylvania State University, University Park, PA 16802, USA \\ ${ }^{2}$ Department of Mechanical Engineering, University of Maryland, College Park, MD 20742, USA \\ ${ }^{3}$ Center for Integrated Nanotechnologies, Sandia National Laboratories, Albuquerque, NM 87185, USA \\ ${ }^{4}$ Division of Engineering, Brown University, Providence, RI 02912, USA \\ Correspondence should be addressed to Sulin Zhang, suz10@psu.edu
}

Received 11 April 2011; Accepted 11 April 2011

Copyright (C) 2011 Sulin Zhang et al. This is an open access article distributed under the Creative Commons Attribution License, which permits unrestricted use, distribution, and reproduction in any medium, provided the original work is properly cited.

Carbon has long been known to exist in three forms: amorphous carbon, graphite, and diamond. However, the discovery of buckyballs in 1985 has created an entirely new branch of carbon chemistry. The subsequent discovery of carbon nanotubes (CNTs) in 1991 has opened up a new era in materials science and nanotechnology. The wonder of the carbon world remains with the successful isolation of monolayer graphene from graphite simply using adhesive tape in 2004. Over the last decades, carbon nanotechnology has been evolved into a truly interdisciplinary field, encompassing chemistry, physics, materials science, and mechanics. The research excitement was largely driven by the unique properties and diverse applications of these lowdimensional carbon nanomaterials. For example, they are promising candidates for nanofillers to strengthen polymeric composites because of their high strength and stiffness; CNTs can be functionalized to serve as nanoscale probes and can be used as a drug delivery system because of their hollow center; nanodiamond has recently found applications in nanomedicine; due to its exceptional electrical properties, graphene may replace silicon as the next-generation electronic materials.

This special issue features research and review articles that cover a wide range of recent progress on the studies of low-dimensional carbon nanomaterials. These studies span from theoretical/computational to experimental efforts, from pristine to defected low-dimensional carbon nanomaterials, from their mechanical and thermal to their electrical properties, and from their synthesis to their patterning and applications of various kinds. Highlighted below are several important contributions from this special issue.

Mechanical properties of low-dimensional carbon nanomaterials continue to be one of the important research topics. Using atomistic simulations, Jun et al. rationalized the underlying physics of the nonlinear elastic softening of monolayer graphene under high pressure. They also predicted that the elastic softening is size dependent. Combining the shear-lag model and fracture mechanics, Liu et al. carried out a hierarchical failure analysis on CNTreinforced composites with hard matrix. Their analyses predicted an optimal interfacial bond density for enhanced fracture toughness. They further predicted that the interface length plays a key role in determining the fracture toughness of the CNT-reinforced composites. This study is particularly useful to the rational design CNT-based composites. Zhang and $\mathrm{Li}$ addressed the morphologic interaction between graphene and $\mathrm{Si}$ nanowires on a $\mathrm{SiO}_{2}$ substrate using molecular mechanics simulations. Their predictive results provide valuable guidance in patterning of graphene sheets on substrates with nanoscale scaffolds. Lu et al. quantified adhesive interactions of small functionalized single-walled CNT (SWCNT) bundles using an atomic force microscopybased adhesive force mapping technique combined with a statistical analysis method. This measurement turns out to be important in the design and fabrication of SWCNTreinforced nanocomposites. Ya'akobovitz et al. developed a geometrically nonlinear string model to analyze the mechanical behavior of SWCNTs under thermoelectrical loading. 
Several articles addressed new synthesis approaches of low-dimensional carbon nanomaterials. Peng et al. fabricated multiwalled carbon nanotube-(MWCNT-)-based circuits and characterized their electrical properties. Yang et al. synthesized onion-like fullerenes by chemical vapor deposition and characterized their ferromagnetic properties. $\mathrm{Li}$ et al. developed a catalyzed carbonization approach with which homogeneous $\mathrm{CNT} /$ carbon composites are fabricated. Lentz et al. synthesized and characterized glassy carbon nanowires. He and Gao developed a novel method to synthesize $\mathrm{Fe}_{3} \mathrm{O}_{4} / \mathrm{Pt}$ nanoparticles-decorated CNTs. These synthesis approaches add new capabilities to the community on making carbon nanomaterials of unique properties.

Novel applications of low-dimensional carbon nanomaterials have been proposed in this special issue. Cao et al. demonstrated the promise of MWCNT-based humidity sensor. Lee et al. proposed that Au-decorated single-walled carbon nanotube field effect transistors can be used as single nucleotide polymorphism detector. He and Gao pointed out that $\mathrm{Fe}_{3} \mathrm{O}_{4} / \mathrm{Pt}$ nanoparticles-decorated CNTs can be used as magnetically recyclable catalysts.

Finally, Mostofizadeh et al. thoroughly reviewed the synthesis, properties, and applications of low-dimensional carbon nanomaterials, ranging and 0-dimensional fullerenes, carbon-encapsulated metal nanoparticles, nanodiamnonds, 1-dimensional carbon nanofibers, CNTs, and 2-dimensional graphene sheets. The review article provided the latest update of the progress in the low-dimensional carbon nanomaterials research and would serve as a convenient resource for the researchers in the field.

We would like to thank the authors across the world for their valuable contributions to this special issue as well as the reviewers for their constructive comments to the manuscripts. We envision that low-dimensional carbon will continue to be one of the promising research fields in the years to come, manifested by the active responses of researchers in the course of this special issue. We are happy to be informed that, owing to the great success of this special issue, Journal of Nanomaterials has decided, for the first time since its launch, to establish an annual/special issue on low-dimensional carbon nanomaterials. We encourage and appreciate your further support for this annual/special issue series. 

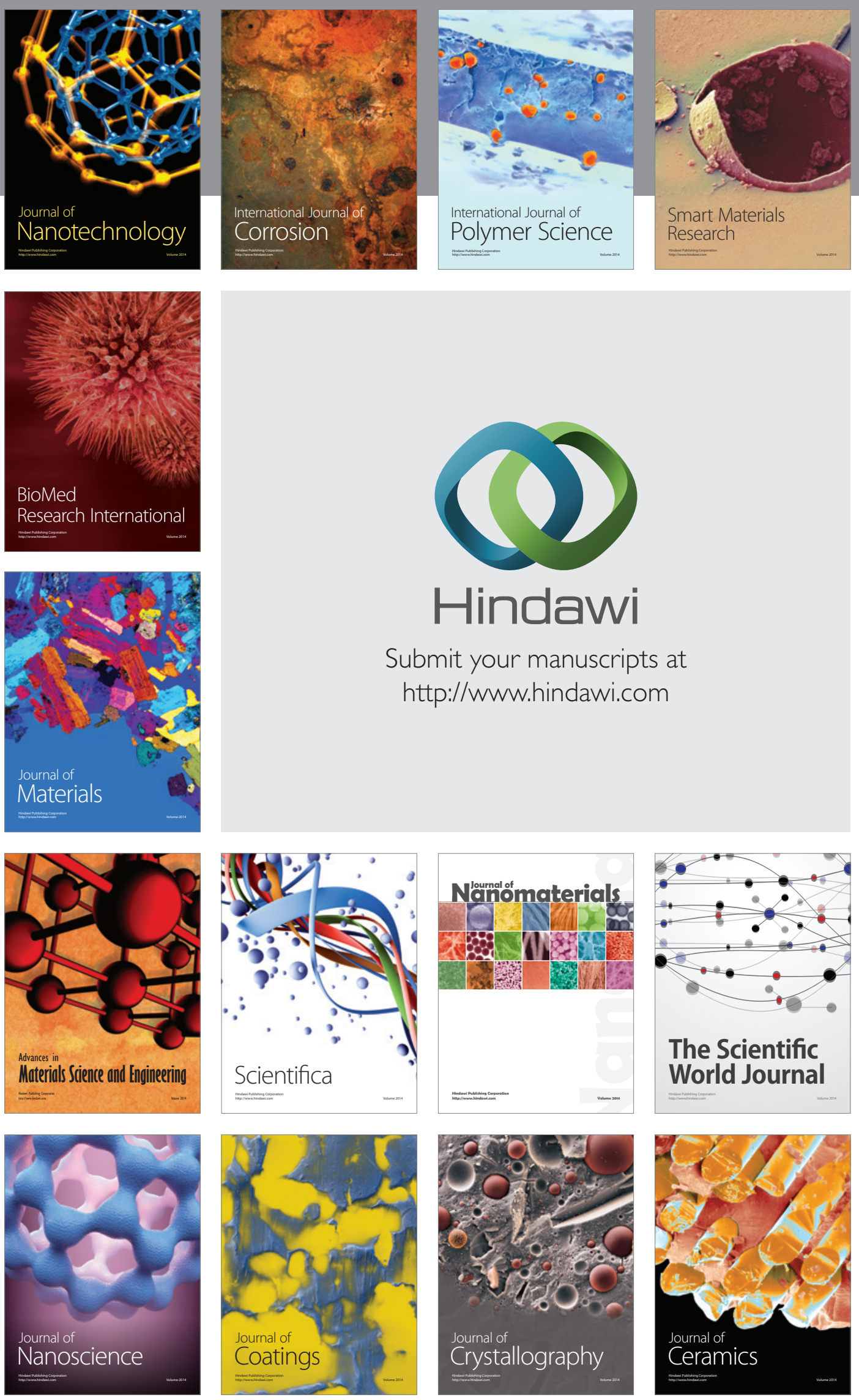

The Scientific World Journal

Submit your manuscripts at

http://www.hindawi.com

\section{World Journal}

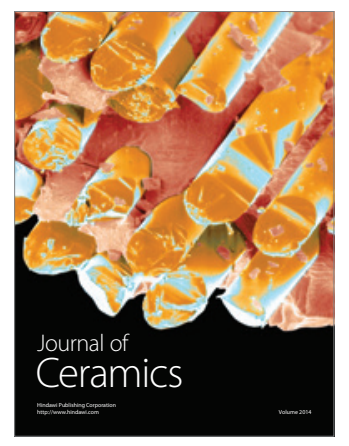

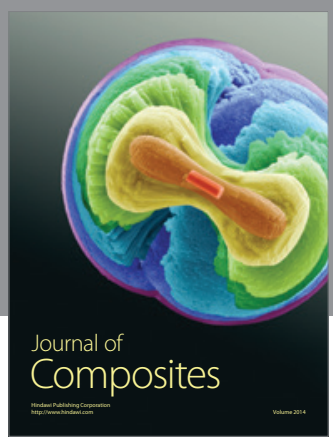
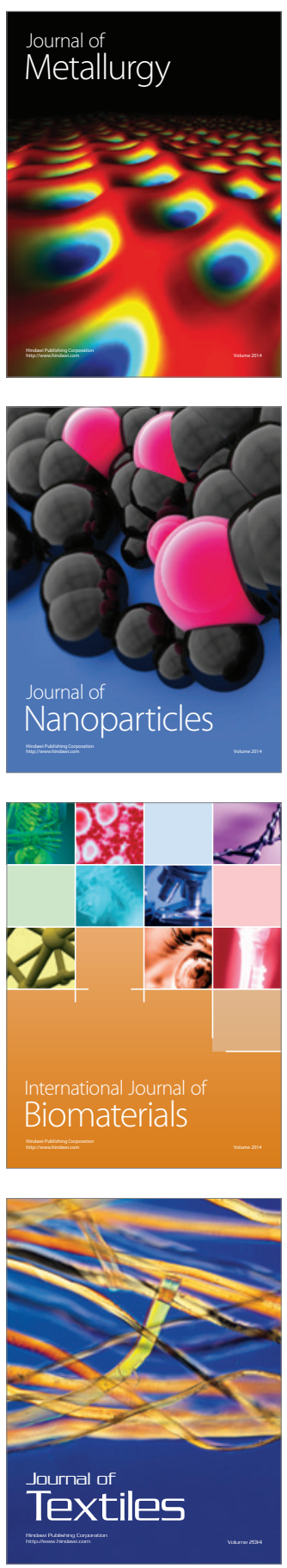